\title{
ACE and ACTN3 genes polymorphisms among female Hungarian athletes in the aspect of sport disciplines
}

\author{
E Bosnyák ${ }^{1}$, E Trájer ${ }^{1}$, A Udvardy ${ }^{1}$, Z Komka ${ }^{1}$, A Protzner ${ }^{1}$, T Kováts ${ }^{1}$, I Györe ${ }^{2}$, \\ M Tóth ${ }^{1}$, J Pucsok ${ }^{3}$, M Szmodis ${ }^{1}$ \\ ${ }^{1}$ Department of Health Sciences and Sports Medicine, University of Physical Education, Budapest, Hungary \\ ${ }^{2}$ Health Centre of Hungarian Defence Force, Kecskemét, Hungary \\ ${ }^{3}$ National Institute for Sports Medicine, Budapest, Hungary
}

Received: March 19, 2014

Accepted: October 7, 2015

\begin{abstract}
The aim of the study was to determine the importance of two sport-associated gene polymorphisms, alpha-actinin-3 R577X (ACTN3) and angiotensin-converting enzyme I/D (ACE), among Hungarian athletes in different sports. The examination was carried out only on women $(n=100)$. Sport-specific groups were formed in order to guarantee the most homogeneous clusters. Human genomic DNA was isolated from blood, and genotyping was performed by polymerase chain reaction. To measure the differences between the participating groups, Chi-squared test was performed using Statistica 9.0 for Windows ${ }^{\circledR}$ (significance level: $p<0.05$ ). In comparing the ACE I/D allele frequencies, significant difference was detected between water polo $(\mathrm{I}=61.11 \%$; $\mathrm{D}=38.89 \%)$ and combat sports $(\mathrm{I}=35.71 \%, \mathrm{D}=64.29 \%)$ athletes $(p<0.03)$. There was no statistical difference when ACE I/D alleles in combat sports and kayaking/rowing $(p>0.05)$ were compared. A similarity was detectable in the I allele frequencies of the water polo (61.11\%) and kayaking/rowing (56.67\%) groups. The ACTN3 R/X polymorphism showed no differences in comparison with the sport groups. $\mathrm{R}$ allele frequencies were higher in every group compared to the $\mathrm{X}$ allele. The potential significance of the ACE I allele in sports of an aerobic nature was not clearly confirmed among Hungarian athletes.
\end{abstract}

Keywords: ACE, ACTN3, gene polymorphisms, elite athletes, physical performance

Nowadays it is well recognized that apart from vigorous training and environmental factors, athletic performance highly depends on the athlete's genetic background. Regarding genetic background, the angiotensin-converting enzyme (ACE) and the $\alpha$-actinin-3 (ACTN3) genes are two of the most widely studied performance-related genes, although their influence on physical performance has not been completely explored $(5,17,22,23)$. The angiotensinconverting enzyme is an important member of the renin-angiotensin system, which converts angiotensin I to angiotensin II. The interaction between angiotensin II and AT1 receptors can cause vasoconstriction, which influences blood pressure. This reaction also generates the release of vasopressin and aldosterone, and water and renal tubular sodium reabsorption and decreases renin level in the blood (11). Furthermore, one polymorphism of the ACE gene supports the energy-depository, enabling the enhancement of metabolic efficiency (16). Due to the crucial role of ACE in cardiovascular homeostasis, this polymorphism can be one part of a possible explanation for the individual variability among the exercise-related phenotypes.

Corresponding author: Edit Bosnyák

Department of Health Sciences and Sports Medicine, University of Physical Education

Alkotás utca 44, H-1123 Budapest, Hungary

Phone: +36 1-487-9200/61188; E-mail: bosnyaked@gmail.com 
The human angiotensin-converting enzyme gene consists of two alleles: insertion (I) allele contains a 287 base pair Alu repeat sequence, and is associated with lower circulating and tissue ACE activity, while deletion (D) allele lacks the Alu repeat sequence and is connected to relatively higher enzyme activity (2).

In the first study describing the connection between ACE polymorphism and elite endurance performance, Gayagay et al. showed that I allele and II genotypes were significantly higher in rowers than in the non-athlete control group (5). In the following years, several other studies reported higher I allele and genotype frequencies in elite endurance athletes. These studies were performed on either mixed-gender groups or male-athlete groups $(2,9)$. A British study showed the effects of a 9-week-long power training program which measured the changes in the quadriceps muscles of 33 healthy volunteers. A significantly higher muscle strength increase was shown among the members possessing D allele (4). Another study, carried out with 99 amateur male athletes, showed a higher muscle power increase connected to DD genotype (1). In a recent Polish study, the I allele frequency was significantly higher in rowers compared to a control group (8).

The $\mathrm{R}$ and $\mathrm{X}$ alleles of the ACTN 3 gene are also candidate polymorphisms that may influence physical performance, especially in the sprint or in power output. The ACTN 3 gene encodes the $\alpha$-actinin-3 protein, which is the dominant isoform in muscle fiber type II, and is responsible for fast, dynamic and powerful muscle contractions (23). In the X allelic variation, the replacement of arginin (R) with a premature stop codon (X) at amino acid 577 results in a nonsense mutation (19). The $\mathrm{X}$ allele is typical in a decisively high percentage of the human population, and signifies the complete absence of the $\alpha$-actinin-3 protein (14). However, the amino acid sequence of the $\alpha$-actinin-2 isoform is similar to the isoform 3 and can be found in both muscle fiber types. This finding suggests that isoform 3 is functionally dispensable, as the $\alpha$-actinin- 2 protein is able to compensate for it and no pathological phenotype evolves (15). Yet, studies on elite athletic performance found a significant difference in ACTN3 genotypes between power and endurance athletes: $\mathrm{R}$ allele was significantly higher in sprint athletes, whereas the $\mathrm{X}$ allele frequency was higher in the endurance group, although the difference was significant only in women (23).

Grenda et al. compared long distance and short distance swimmers with control subjects and no significant association was found for ACTN3 polymorphism (7).

Following Yang's examination, several studies have dealt with significantly lower XX genotypes among sprinters and power athletes compared to endurance athletes $(18,20,22)$.

There is still a discrepancy in the results (especially in the case of the ACE I/D polymorphism); the alternating grouping system of the athletes makes it impossible to compare the already published essays. The meta-analysis of Ma et al. included more than 400 articles dealing with ACE or ACTN3 polymorphisms (13). The study - based on the results of 48 selected relevant articles - revealed more solid evidence of connections between the ACE II genotype and endurance performance, as well as between ACTN3 R allele and power events. Standard or sport-specific grouping is needed for greater homogeneity and easier comparison. Clustering based on the sports could be one of the most straightforward ways to create homogeneous groups. Ma et al. also emphasized the importance of standardizing the criteria of elite athletes (13). Sport-specific groups were created and compared to each other with the aim of showing the possible differences in the genetic background of athletes in different fields of sport. Only women were examined in order to clarify the connection between sport performance and individual genetic background (specifically the ACTN3 R577X and ACE I/D genes). According to several internationally recognized past researches and studies, gender differentiation is relevant and can lead to more precise academic results (23). 


\section{Materials and Methods}

\section{Subjects}

The Hungarian Ethics Committee approved the experimental procedures and each individual gave their written informed consent to participate in the study. Athletes from Hungarian national teams participated in this study, including several Olympic and World champions. These individuals won altogether ten Olympic gold medals and more than 100 World Championships. In order to create homogeneous groups, and due to continually improving international results, our attention turned to women. One-hundred (100) female athletes were involved in the examination, representing seven different sports: kayaking, rowing, judo, wrestling, handball, basketball and water polo. Four groups were established based on the metabolism of the sports and the characteristic of performance: combat sports (judo, wrestling, $n=14)$; handball/basketball $(n=29)$; water polo $(n=27)$; kayaking/rowing $(n=30)$.

\section{Genotyping}

Human genomic DNA was isolated from blood by phenol-chloroform extraction following cell lysis with Triton X-100 and digestion with Proteinase K. PCR products were separated on $4 \%$ agarose gel and became visible through ultraviolet light (Ultra Lum transilluminator, Fuji digital photohead, Adobe PhotoDeluxe v 4.0) after $2 \mu$ dropped ethidium bromid (Sigma) $(16,21)$.

\section{Statistical analysis}

The allele frequency differences among the groups were calculated with Pearson's chisquared test analysis, which was performed using Statistica 9.0 for Windows ${ }^{\circledR}$ (Statsoft Inc., Tulsa, OK, USA).

The Chi-square test was also used to compare the allele frequencies of the different groups, since the Hardy-Weinberg equilibrium had already been estimated. In appropriate cases Fisher's exact test was also used. Statistical significance level was defined as $p<0.05$.

\section{Results}

ACE I/D genotype distributions [II (28\%), ID (48\%), DD (24\%); I allele frequency (0.52)] were consistent with Hardy-Weinberg equilibrium (Fig. 1). In comparing the alleles of the combat-sport group to the water polo group, we found a significant difference $\left(\chi^{2}=4.77, p<\right.$ 0.03 ) (Fig. 2). The difference of the allele frequencies in the kayaking/rowing group compared to that of combat sports was not significant $\left(\chi^{2}=3.35, p>0.05\right)$ (Fig. 3). The allele frequencies of the water polo and kayaking/rowing groups showed a similar ratio (Fig. 4). There was no significant difference found in a comparison of the handball/basketball group to the other groups (kayaking/rowing: $\chi^{2}=1.21, p>0.05$; combat sports: $\chi^{2}=0.90, p>0.05$; water polo: $\left.\chi^{2}=2.38 p>0.05\right)$.

ACTN3 R/X genotype frequencies were also in Hardy-Weinberg equilibrium. No statistical differences between the groups (Fig. 5) were found either in genotype distributions or in allelic frequencies. The R allele frequency was higher than the frequency of the $\mathrm{X}$ allele in each group. 


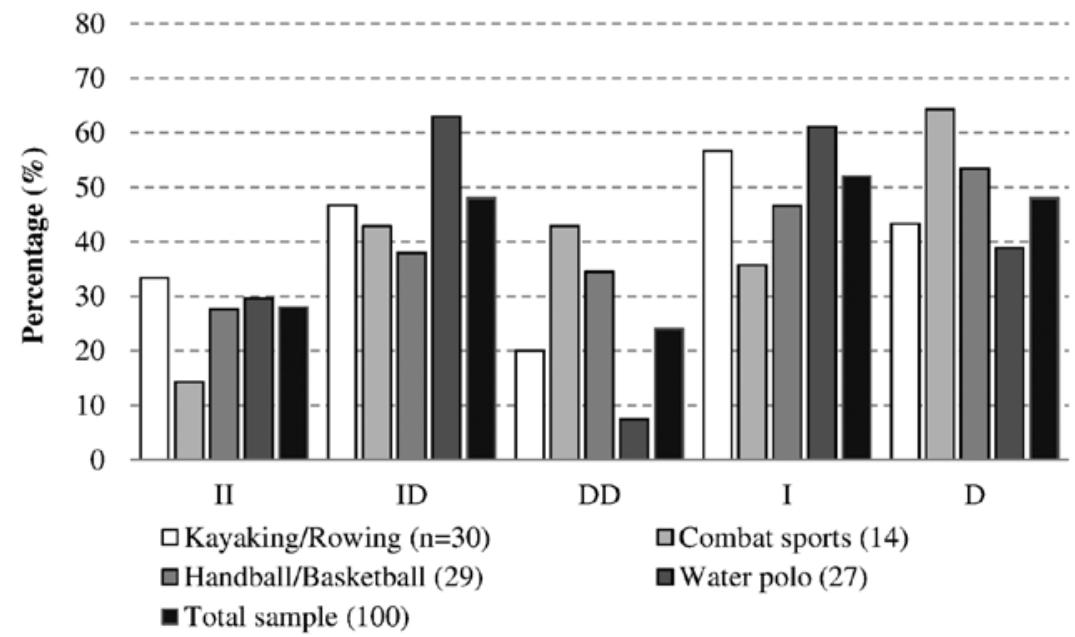

Fig. 1. ACE genotypes and allele frequencies in 100 athletes compared by groups

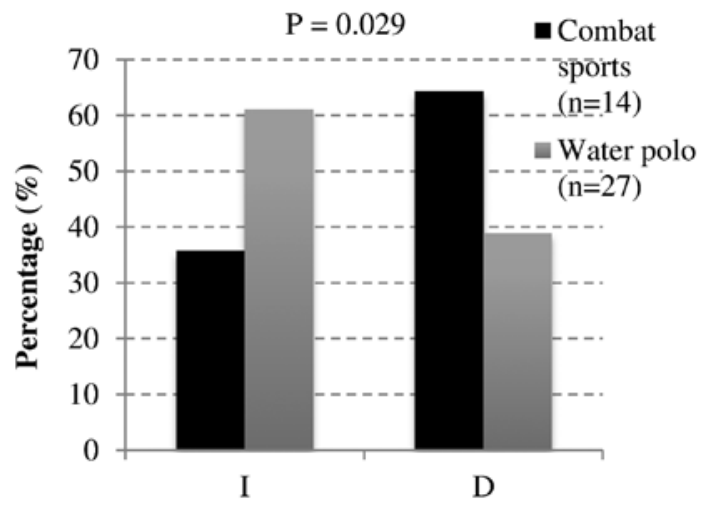

Fig. 2. Differences in the ACE allele frequencies in combat sports and water polo

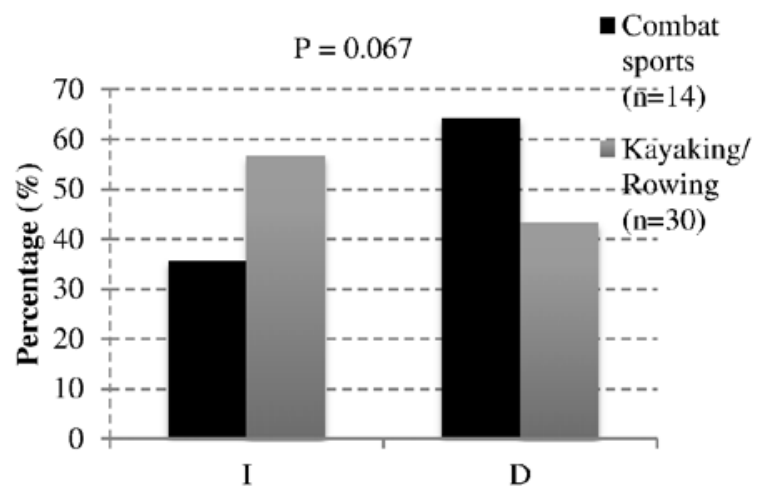

Fig. 3. The ACE allele frequencies in combat sports and kayaking/rowing 


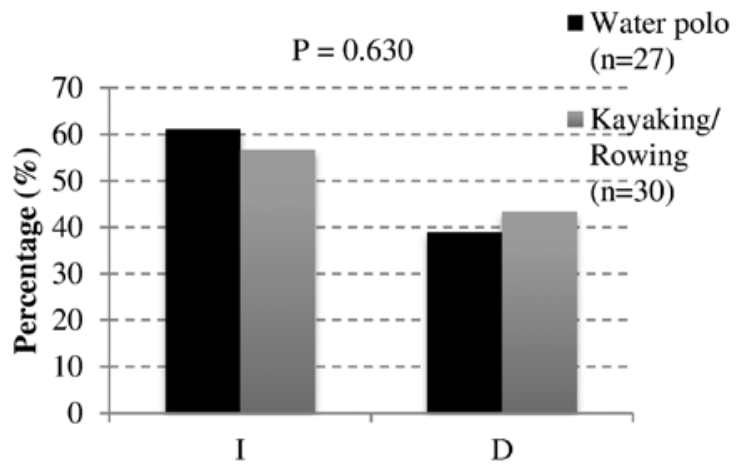

Fig. 4. Similarity in the ACE allele frequencies in water polo and kayaking/rowing

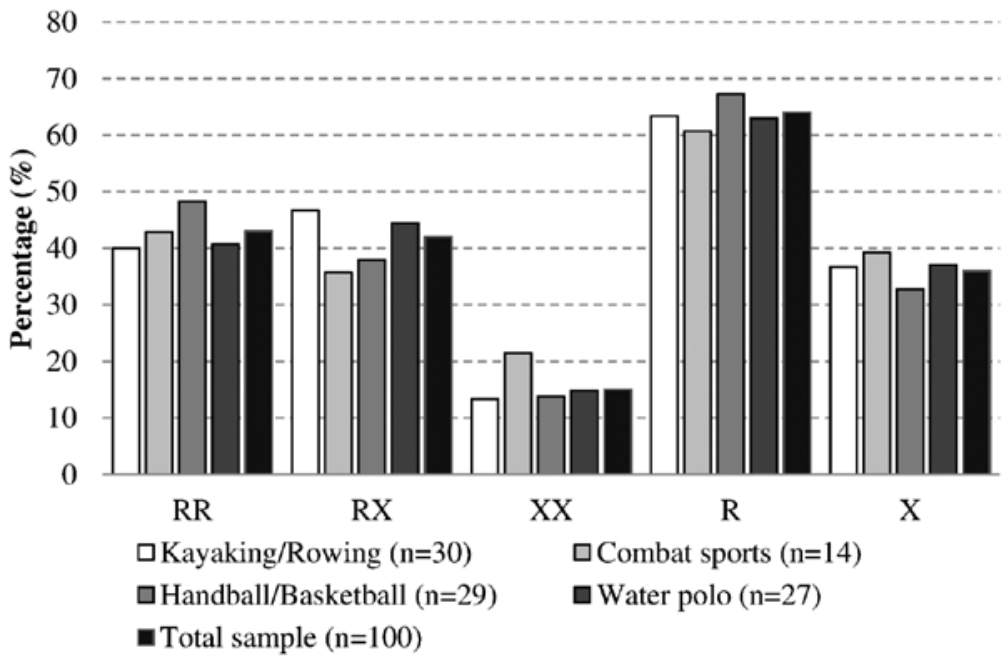

Fig. 5. ACTN3 genotypes and allele frequencies in 100 athletes, compared by groups

\section{Discussion}

Increased occurrence of the ACE D allele was noticed for combat sports (64.29\%), while the frequency of the ACE II genotype was considerably low (14.29\%); supposedly, there can be a connection to the more significant anaerobic features of combat sports. These results fulfilled our expectations, although a Lithuanian study could display neither a higher D allele frequency nor lower II genotype occurrence among the athletes integrated into the power or mixed group (6).

Our hypothesis was justified as the I allele was higher $(56.67 \%)$ than the D allele $(43.33 \%)$ in kayaking and rowing, but there was no significant difference between the frequencies. In an Australian investigation concerning rowers, the same results were detected (I allele: 57\%; D allele: 43\%) and the genotype frequencies were similar as well (16). Also, in a recent Polish study with 121 male rowers the same tendency was detectable. The I allele frequency was significantly higher in rowers, compared to a control group (8). 
The ACE allele-frequencies differed significantly between water polo and combat sports groups $(p<0.03)$. This difference might be related to the difference in energy utilization. In the water polo group, the ID genotype $(62.96 \%)$ was conclusively higher than the other two genotypes. Similar results were found in a study with Lithuanian athletes (6). With regard to the "mixed athletes" group (both anaerobic and aerobic energy production), the percentage of ID genotype was $47.70 \%$. Most of our water polo related results matched those of the "power oriented group" in which the genotype prevalence was $26 \%, 57 \%$, and $19 \%$, respectively, for II, ID, and DD.

The genotype frequencies among the handball/basketball players did not differ $(\mathrm{I}=$ $47 \%, \mathrm{D}=53 \%$ ). Female team players and individual athletes were examined separately in a study from Taiwan (12). The results did not show a difference between the genotype frequencies of the two groups. The occurrence of I allele was definitely higher $(64 \%)$ than that of the D allele (38\%). In our study there was no significant difference between the two polymorphisms and in spite of the results of the Taiwanian study, the frequency of the D allele was higher. If we compare the water polo group to the team sports group from the Taiwanese study, we can find the same results. The exact sports included in the Taiwanese study were not mentioned. This ambiguity between the results of the team sport groups has to be noted in order to understand the reasons for the grouping of the athletes by sport. The findings may not be comparable in any other way, and may lead to the strong probability that incorrect conclusions can be drawn from them. In the case of sports that are determined by anaerobic lactic procedures (combat sports, ball games), the D allele frequency was more than $50 \%$, whereas for endurance-based disciplines the I allele occurrence was higher.

With regard to the ACTN3 gene, in the group of kayaking/rowing and water polo, a similarity was detectable between the polymorphisms, since the role played by power is just as determining as that played by endurance. The RR genotype distribution percentage of Israeli sprinters was $52 \%$ and the $\mathrm{R}$ allele frequency was $70 \%$ (3). These results resembled those in our handball/basketball group, which was unexpected, since these two sports combine sprint, power and endurance output as well; they are not only sprint specific. In comparing the handball/basketball group's ACTN3 polymorphisms to those of the Taiwanese study's team players, we could not find a similarity (12). The same applied to the water polo group's genotype distribution.

Among combat sport athletes the XX genotype was higher than expected. In an Indian study, among power athletes the XX genotype was underrepresented (10). In another study from the United States the XX genotype distribution was significantly lower among strength athletes $(6.70 \%)$ compared to the control group (16.30\%) (22). Combat sports are considered to be power endurance sports, so the distribution of the XX genotype may not be very significant.

There were no differences in the handball/basketball group compared to any other examined group in any polymorphism of the ACE and the ACTN3 genes. This can provide the basis for several motoric ability that is necessary to perform these sports at an outstanding level. It is interesting that we failed to demonstrate an ACTN3 R allele frequency difference between the four groups while the examined athletes' sports differ from each other in many aspects (metabolism, muscle performance, conditional, coordination skills, etc.). The lack of an association between the ACTN3 genotype and different sports may lead researchers to the conclusion that the sport-based differentiation of this genotype should be a priority. 
These results show that mixed cohort grouping can be misleading in terms of defining the connections between polymorphisms and elite performance. The potential significance of the ACE I allele in sports of an aerobic nature was not clearly confirmed with the Hungarian athletes, even if the results show the dominant presence of it.

A comparison of the athletes from different sports to a control group would be important from the aspect of researchers being able to draw better defined conclusions. The type of genetic background can be important for an athlete to be able to continuously perform at a high level, but may not be that essential in every sport, especially not in the case of sports which require the complexity of various sporting skills. For further conclusive deductions, more sport-associated polymorphisms must be included in examinations in order to clarify inter-gene connections. Another potential research field could be the examination of special physical performance parameters in connection with a selected polymorphism.

\section{Acknowledgements}

Financial support: Cardio 08, TÁMOP.4.2.1.B-09/1/ KMR-2010-0001; Új Széchenyi Terv; KTA_AIK-12-1-20130017 and GOP-1.1.1-2012-0258.

\section{REFERENCES}

1. Colakoglu M, Sirrı Cam F, Kayitken B, Cetinoz F, Colakoglu S, Turkmen M, Sayin M: ACE genotype may have an effect on single vs. multiple-set preferences in strength training. Eur. J. App. Physiol. 95, 20-26 (2005)

2. Danser AH, Schalekamp MA, Bax WA, Van den Brink AM, Saxena PR, Riegger GA, Schunkert H: Angiotensinconverting enzyme in the human heart. Effect of the deletion/insertion polymorphism. Circulation 92, 13871388 (1995)

3. Eynon N, Duarte JA, Oliveira J, Sagiv M, Yamin C, Meckel Y, Goldhammer E: ACTN3 R577X polymorphism and Israeli top-level athletes. Int. J. Sports Med. 30, 695-698 (2009)

4. Folland J, Leach B, Little T, Hawker K, Myerson S, Montgomery H, Jones D: Angiotensin-converting enzyme genotype affects the response of human skeletal muscle to functional overload. Exp. Physiol. 85, 575-579 (2000)

5. Gayagay G, Yu B, Hambly B, Boston T, Hahn A, Celermajer DS, Trent RJ: Elite endurance athletes and the ACE I allele: the role of genes in athletic performance. Hum. Genet. 103, 48-50 (1998)

6. Ginevičienė V, Pranculis A, Jakaitienė A, Milašius K, Kučinskas V: Genetic variation of the human ACE and ACTN3 genes and their association with functional muscle properties in Lithuanian elite athletes. Medicina (Kaunas) 47, 284-290 (2011)

7. Grenda A, Leońska-Duniec A, Kaczmarczyk M, Ficek K, Król P, Cięszczyk P, Żmijewski P: Interaction between ACE I/D and ACTN3 R557X polymorphisms in Polish competitive swimmers. J. Hum. Kinet. 42, 127-136 (2014)

8. Jastrzebski Z, Leonska-Duniec A, Kolbowicz M, Tomiak T: The angiotensin converting enzyme gene I/D polymorphism in Polish rowers. Centr. Eur. J. Sport Sci. Med. 5, 77-82 (2014)

9. Juffer P, Furrer R, Gonzalez-Freire M, Santiago C, Verde Z, Serratosa L, Morate FJ, Rubio JC, Martin MA, Ruiz JR, Arenas J, Gomez-Gallego F, Lucia A: Genotype distributions in top-level soccer players: a role for ACE? Int. J. Sports Med. 30, 387-392 (2009)

10. Kothari ST, Chheda P, Chawla S, Chatterjee L, Chaudhry SK, Das BR: ACTN3 R577X polymorphism in Asian Indian athletes. Int. J. Hum. Genet. 11, 149-153 (2011)

11. Lavoie JL, Sigmund CD: Minireview: overview of the renin-angiotensin system-an endocrine and paracrine system. Endocrinology144, 2179-2183 (2003)

12. Li-Ling C, Ling-Ling H, Ke-Tien Y, Sandy SH: ACE I/D and ACTN3 R577X polymorphism in elite athletes. Med. Sci. Sports Exerc. 37, 167 (2005) 
13. Ma F, Yang Y, Li X, Zhou F, Gao C, Li M, Gao L: The association of sport performance with ACE and ACTN3 genetic polymorphisms: a systematic review and meta-analysis. PLoS One 8, e54685 (2013)

14. MacArthur DG, North KN: A gene for speed? The evolution and function of $\alpha$-actinin-3. BioEssays 26, 786-795 (2004)

15. Mills M, Yang N, Weinberger R, Vander Woude DL, Beggs AH, Easteal S, North K: Differential expression of the actin-binding proteins, alpha-actinin-2 and -3 , in different species: implications for the evolution of functional redundancy. Hum. Mol. Genet. 10, 1335-1346 (2001)

16. Montgomery HE, Clarkson P, Barnard M, Bell J, Brynes A, Dollery C, Hajnal J, Hemingway H, Mercer D, Jarman P, Marshall R, Prasad K, Rayson M, Saeed N, Talmud P, Thomas L, Jubb M, World M, Humphries S: Angiotensin-converting-enzyme gene insertion/deletion polymorphism and response to physical training. Lancet 353, 541-545 (1999)

17. Myerson S, Hemingway H, Budget R, Martin J, Humphries S, Montgomery H: Human angiotensin I-converting enzyme gene and endurance performance. J. Appl. Physiol. 87, 1313-1316 (1999)

18. Niemi AK, Majamaa K: Mitochondrial DNA and ACTN3 genotypes in Finnish elite endurance and sprint athletes. Eur. J. Hum. Genet. 8, 965-969 (2005)

19. North KN, Yang N, Wattanasirichaigoon D, Mills M, Easteal S, Beggs AH: A common nonsense mutation results in alphaactinin-3 deficiency in the general population. Nat. Genet. 21, 353-354 (1999)

20. Papadimitriou ID, Papadopoulos C, Kouvatsi A, Triantaphyllidis C: The ACTN3 gene in elite Greek track and field athletes. Int. J. Sports Med. 4, 352-355 (2008)

21. Rigat B, Hubert C, Corvol P, Soubrier F: PCR detection of the insertion/deletion polymorphism of the human angiotensin converting enzyme gene (DCP1) (dipeptidyl carboxypeptidase 1). Nucleic Acids Res. 20, 1433 (1992)

22. Roth SM, Walsh S, Liu D, Metter EJ, Ferrucci L, Hurley BF: The ACTN3 R577X nonsense allele is underrepresented in elite-level strength athletes. Eur. J. Hum. Genet. 16, 391-394 (2008)

23. Yang N, MacArthur DG, Gulbin JP, Hahn AG, Beggs AH, Easteal S, North K: ACTN3 genotype is associated with human elite athletic performance. Am. J. Hum. Genet.73, 627-631 (2003) 